PROCEEDINGS OF THE AMERICAN MATHEMATICAL SOCIETY

Volume 124, Number 8, August 1996

\title{
A SMALL DOWKER SPACE IN ZFC
}

\author{
ZOLTAN T. BALOGH \\ (Communicated by Franklin D. Tall)
}

\begin{abstract}
We construct a hereditarily normal topological space whose product with the unit interval is not normal. The space is $\sigma$-relatively discrete and has cardinality of the continuum $\mathfrak{c}$.
\end{abstract}

Introduction. A Dowker space is a normal Hausdorff space whose product with the closed unit interval $I$ is not normal. Whether there are such spaces at all was raised as a problem by C. H. Dowker [D] in 1951 who also gave an internal characterization of Dowker spaces. (See Lemma 0.1 below.)

Constructing Dowker spaces turned out to be a hard, many-faceted problem in general and set-theoretic topology, of basically combinatorial set-theoretic nature. It has extensive, rich literature. (See the survey paper $\left[\mathrm{R}_{4}\right]$ and also $\left[\mathrm{R}_{6}\right],\left[\mathrm{W}_{2}\right]$ for some update.) There has been precisely one Dowker space built in usual (ZFC) mathematics by Mary Ellen Rudin $\left[\mathrm{R}_{2}\right]$ in 1971 . This space has cardinality and weight $\aleph_{\omega}^{\omega}$, and it has few nice properties beyond normality. The search for a smaller example is referred to as the Small Dowker Space Problem. The effort of a number of mathematicians resulted in nice, small Dowker spaces in many models of set theory $[\mathrm{Be}],[\mathrm{C}],[\mathrm{G}],[\mathrm{JKR}],\left[\mathrm{R}_{1}\right],\left[\mathrm{R}_{5}\right]$, [We], but no example different from Rudin's has been constructed in ZFC alone.

In this paper we give a solution to the Small Dowker Space Problem by constructing, without extra set-theoretic axioms, a hereditarily normal, $\sigma$-relatively discrete Dowker space of cardinality of the continuum $\mathfrak{c}$. Such a space of strongly compact cardinality (under the set-theoretic assumption that such large cardinals exist), was constructed by S. Watson $\left[\mathrm{W}_{1}\right]$. Our proof uses a general combinatorial technique originated in M. E. Rudin's $\left[\mathrm{R}_{3}\right]$ and later used by the author $[\mathrm{B}]$ to construct Q-set spaces.

Our terminology and notation follow the standards of contemporary set theory and set-theoretic topology as used in $[\mathrm{K}]$ and $[\mathrm{KV}]$. In particular, $[A]^{<\kappa}$ stands for the family of all subsets of $A$ of cardinality $<\kappa$ and $F n(\kappa, 2)$ denotes all finite partial functions from $\kappa$ to $2=\{0,1\}$.

We shall make use of Dowker's internal characterization.

Lemma 0.1. The following conditions are equivalent for a normal $T_{1}$ space $X$.

(a) $X$ is a Dowker space.

Received by the editors March 23, 1994.

1991 Mathematics Subject Classification. Primary 54D15, 54D20.

Key words and phrases. Small Dowker space, hereditarily normal, elementary submodel.

Research supported by NSF Grant DMS - 9108476.

(C)1996 American Mathematical Society 
(b) $X$ is not countably metacompact, i.e., there is an increasing open cover $G_{0} \subset$ $G_{1} \subset \ldots \subset G_{n} \subset \ldots, n \epsilon \omega$, of $X$ such that for every sequence of closed sets $F_{n} \subset G_{n}, \cup_{n \epsilon \omega} F_{n} \neq X$.

\section{The space.}

Theorem 1.1. There is a hereditarily normal, $\sigma$-relatively discrete Dowker space of cardinality $\mathfrak{c}$.

The heart of the proof of Theorem 1.1 is the following combinatorial lemma.

Lemma 1.2. Let $\lambda=2^{\mathfrak{c}}$, and let $\left\langle c_{\xi}\right\rangle_{\xi<\lambda}$ be a one-to-one enumeration of ${ }^{\mathfrak{c}} 2=\{c: c$ is a function from $\mathfrak{c}$ to 2$\}$. Then there is a sequence $\left\langle d_{\xi}\right\rangle_{\xi<\lambda}$ of functions $d_{\xi}: \mathfrak{c} \rightarrow 2$ in such a way that for every $g: \mathfrak{c} \rightarrow[\lambda]^{<\omega}, f: \mathfrak{c} \rightarrow \omega$ and $h: \mathfrak{c} \rightarrow[\mathfrak{c}]^{<\omega}$, there are $\alpha<\beta$ in $\mathfrak{c}$ such that $f(\alpha)=f(\beta), \beta \notin h(\alpha)$ and for every $\xi \in g(\alpha), c_{\xi}(\alpha)=d_{\xi}(\beta)$.

Proof of Lemma 1.2. Let us call a triple $\langle A, B, u\rangle$ a control triple if

(1) $A \in[\mathfrak{c}]^{\omega}, B \in\left[{ }^{A} 2\right] \leq \omega$

(2) $u$ is a function with $\operatorname{dom}(u) \epsilon[A]^{\omega}$;

(3) for every $\alpha \epsilon \operatorname{dom}(u), u(\alpha) \epsilon\left[{ }^{A} 2 \backslash B\right]^{<\omega}$;

(4) if $\alpha \neq \alpha^{\prime}$ in $\operatorname{dom}(u)$, then $u(\alpha) \cap u\left(\alpha^{\prime}\right)=\emptyset$.

Let $\left\langle A_{\beta}, B_{\beta}, u_{\beta}\right\rangle_{\beta<\mathfrak{c}}$ be a list of all control triples mentioning each triple $\mathfrak{c}$ many times.

Suppose now that $\xi<\lambda$ and we want to define $d_{\xi}(\beta)$ for some $\beta<\mathfrak{c}$. We are going to distinguish among three cases.

Case 1. If $c_{\xi} \uparrow A_{\beta} \in B_{\beta}$, then let $d_{\xi}(\beta)=c_{\xi}(\beta)$.

Case 2. If $c_{\xi}\left\lceil A_{\beta} \epsilon u_{\beta}(\alpha)\right.$ for some $\alpha \epsilon \operatorname{dom}\left(u_{\beta}\right)$, then note first that $c_{\xi}\left\lceil A_{\beta} \notin\right.$ $B_{\beta}$ by (3), and that there is only one such $\alpha$ by (4). Then define $d_{\xi}(\beta)=c_{\xi}(\alpha)$.

Case 3. If neither Case 1 nor Case 2 holds, then set $d_{\xi}(\beta)=0$.

The rest of the proof of Lemma 1.2 will be devoted to showing that the sequence $\left\langle d_{\xi}\right\rangle_{\xi<\lambda}$ of functions constructed above satisfies the requirements in the conclusion of Lemma 1.2. To see this, take a $g: \mathfrak{c} \rightarrow[\lambda]^{<\omega}$, an $f: \mathfrak{c} \rightarrow \omega$ and an $h: \mathfrak{c} \rightarrow[\mathfrak{c}]^{<\omega}$. For every $\alpha \epsilon \mathfrak{c}$, let $e_{\alpha} \epsilon F n(\lambda, 2)$ be such that $\operatorname{dom}\left(e_{\alpha}\right)=g(\alpha)$ and that $e_{\alpha}(\xi)=c_{\xi}(\alpha)$ for every $\xi \epsilon g(\alpha)$.

We will produce a pair $\alpha<\beta$ in $\mathfrak{c}$ such that

(5) $f(\alpha)=f(\beta), \beta \notin h(\alpha)$ and for every $\xi \in g(\alpha), c_{\xi}(\alpha)=d_{\xi}(\beta)$.

To do this, fix two countable elementary submodels $M$ and $N$ of $H\left(\left(2^{2^{\mathfrak{c}}}\right)^{+}\right)$such that $\left\langle c_{\xi}\right\rangle_{\xi<\lambda},\left\langle d_{\xi}\right\rangle_{\xi<\lambda},\left\langle e_{\alpha}\right\rangle_{\alpha<\mathfrak{c}, g}, f, h \in M$ and $M \in N$.

Let $A=\mathfrak{c} \cap N, B=\left\{c_{\xi} \mid A: \xi \in \lambda \cap M\right\}$ and let us pick a partial function $u: A \rightarrow\left[{ }^{\left.A_{2} \backslash B\right]^{<\omega}}\right.$ with the following property:

(6) whenever $v \in N$ is an infinite partial function $v: \mathfrak{c} \rightarrow[\lambda \backslash M]^{<\omega}$ and $\alpha \neq$ $\alpha \prime$ in $\operatorname{dom}(v)$ implies $v(\alpha) \cap v(\alpha \prime)=\emptyset$, then there is an $\alpha \epsilon \operatorname{dom}(u) \cap \operatorname{dom}(v) \operatorname{such}$ that

$$
u(\alpha)=\left\{c_{\xi}\lceil A: \xi \in v(\alpha)\} .\right.
$$

To see that there is such a $u$, let $\left\langle v_{j}\right\rangle_{j \epsilon \omega}$ list each $v$ as in (6). Take $\alpha_{0}, \alpha_{1}, \ldots$, $\alpha_{j}, \ldots \epsilon A$ in such a way that $\alpha_{j} \epsilon \operatorname{dom}\left(v_{j}\right) \cap N$ and $i<j \operatorname{implies} v_{i}\left(\alpha_{i}\right) \cap v_{j}\left(\alpha_{j}\right)=\emptyset$. Then let $\operatorname{dom}(u)=\left\{\alpha_{j}: j \in \omega\right\}$, and set $u\left(\alpha_{j}\right)=\left\{c_{\xi} \uparrow A: \xi \epsilon v\left(\alpha_{j}\right)\right\}$ for every $j \in \omega$. We have to show that $u$ satisfies (2), (3) and (4). It obviously satisfies (2). 
To see that (3) is satisfied, suppose indirectly that $u\left(\alpha_{j}\right) \cap B \neq \emptyset$ for some $j \epsilon \omega$, i.e. there are $\xi \epsilon v_{j}\left(\alpha_{j}\right) \subset N$ and $\eta \epsilon \lambda \cap M \subset N$ such that $c_{\xi}\left\lceil A=c_{\eta}\lceil A\right.$. Then $N \models c_{\xi}=c_{\eta}$, so $c_{\xi}=c_{\eta}$. Since $\left\langle c_{\xi}\right\rangle_{\xi<\lambda}$ is a one-to-one enumeration, it follows that $\xi=\eta$, contradicting $v_{j}\left(\alpha_{j}\right) \subset \lambda \backslash M$. The proof that $u$ satisfies (4) is similar.

Returning to finding $\alpha$ and $\beta$ as required in (5), let us pick $\beta<\mathfrak{c}$ to be such that $\beta>\sup A$ and $\left\langle A_{\beta}, B_{\beta}, u_{\beta}\right\rangle=\langle A, B, u\rangle$. To find $\alpha$, let $E=g(\beta) \cap M$ and $e=e_{\beta}\left\lceil E=e_{\beta}\left\lceil(\lambda \cap M)\right.\right.$. Set $n=f(\beta)$. Let us say that $\left\langle e_{\gamma}\right\rangle_{\gamma \epsilon D}(D \subset \mathfrak{c})$ forms a $\triangle$-system with root $e$ iff $e_{\gamma}$ extends $e$ for every $\gamma \in D$, and $\operatorname{dom}\left(e_{\gamma}\right) \cap \operatorname{dom}\left(e_{\delta}\right)=E$ for every $\gamma \neq \delta$ in $D$. Let us take a maximal $D \subset f^{\leftarrow}(\{n\})$ such that $\left\langle e_{\gamma}\right\rangle_{\gamma \in D}$ forms a $\triangle$-system with root $e$. Since $\left\langle e_{\gamma}\right\rangle_{\gamma \epsilon \mathfrak{c}}, f, e \in M$, we can assume that we have taken such a $D \in M$. Then $D$ is uncountable, or else adding $\beta$ to $D$ would contradict maximality. Thus the set

$$
H=\left\{\gamma \in D:\left(\operatorname{dom}\left(e_{\gamma}\right) \backslash E\right) \cap(\lambda \cap M)=\emptyset\right\} \in N
$$

is uncountable. Let $v: H \rightarrow[\lambda \backslash M]^{<\omega}$ be defined by

$$
v(\gamma)=\operatorname{dom}\left(e_{\gamma}\right) \backslash E .
$$

Then $v \epsilon N$, and thus there is an $\alpha \epsilon \operatorname{dom}(u) \cap \operatorname{dom}(v)$ such that

$$
u(\alpha)=\left\{c_{\xi}\lceil A: \xi \in v(\alpha)\} .\right.
$$

This $\alpha$ will satisfy (5) with our $\beta$. Indeed, $\alpha \epsilon D \subset f \leftarrow(\{n\})$, so $f(\alpha)=n=f(\beta)$. Since $\alpha, h \in N$, it follows that $h(\alpha) \subset N$. By $\beta>\sup A=\sup (\mathfrak{c} \cap N)$, we conclude $\beta \notin h(\alpha)$. Finally, let $\xi \in g(\alpha)=\operatorname{dom}\left(e_{\alpha}\right)$. We will distinguish between two cases.

Case A. If $\xi \epsilon E$, then $c_{\xi}(\alpha)=e_{\alpha}(\xi)=e(\xi)=e_{\beta}(\xi)=c_{\xi}(\beta)$ and by $\xi \epsilon E \subset$ $\lambda \cap M$ we have $c_{\xi}\left\lceil A_{\beta}=c_{\xi} \backslash A \epsilon B=B_{\beta}\right.$. Thus by Case 1 of the definition of $d_{\xi}(\beta), d_{\xi}(\beta)=c_{\xi}(\beta)=c_{\xi}(\alpha)$.

Case B. If $\xi \epsilon \operatorname{dom}\left(e_{\alpha}\right) \backslash E=\operatorname{dom} v(\alpha)$, then $c_{\xi}\left\lceil A_{\beta}=c_{\xi}\left\lceil A \epsilon u(\alpha)=u_{\beta}(\alpha)\right.\right.$. Thus by Case 2 of the definition of $d_{\xi}(\beta)$, it follows that $d_{\xi}(\beta)=c_{\xi}(\alpha)$.

The rest of the proof of Theorem 1.1 is similar to the proof of Lemma 1 in $\left[\mathrm{W}_{1}\right]$ of M. E. Rudin and S. Watson.

The rest of the proof of Theorem 1.1. Let $X=\mathfrak{c} \times \omega$. Set, for every $n \epsilon \omega, X_{n}=$ $\mathfrak{c} \times\{n\}$ and $G_{n}=\mathfrak{c} \times(n+1)$. To define the topology on $X$, let us first define, for every $\alpha \epsilon \mathfrak{c}, s \in[\lambda]^{<\omega}$ and $a \epsilon[\mathfrak{c}]^{<\omega}$, the set

$$
F(\alpha, s, a)=\left\{\beta \epsilon \mathfrak{c}: \text { for every } \xi \epsilon s, d_{\xi}(\beta)=c_{\xi}(\alpha)\right\} \backslash a .
$$

Note that for every $\alpha \epsilon \mathfrak{c}$,

$$
\mathfrak{F}_{\alpha}=\left\{F(\alpha, s, a): s \in[\lambda]^{<\omega}, a \epsilon[\mathfrak{c}]^{<\omega}\right\}
$$

is closed under finite intersections. Now, for $x=\langle\alpha, n\rangle \epsilon X$, let

$$
\mathfrak{N}_{x}=\left\{\begin{array}{l}
\{\{x\}\}, \text { if } n=0 ; \\
\left\{\{x\} \cup F \times\{n-1\}: F \in \mathfrak{F}_{\alpha}\right\}, \text { if } n \geq 1 .
\end{array}\right.
$$

Let us now declare a subset $U$ of $X$ to be open if and only if for every $x \epsilon U$, there is an $N \in \mathfrak{N}_{x}$ such that $N \subset U$. Since each $\mathfrak{N}_{x}$ is closed under finite intersections 
and $\cap \mathfrak{N}_{x}=\{x\}$ for every $x \in X$, this defines a $T_{1}$ topology $\tau$. Clearly, each $X_{n}$ is a discrete subspace of $X$ (equipped with the topology $\tau$ ), so $X$ is a $\sigma$-relatively discrete $T_{1}$ space.

To see that $X$ is hereditarily normal, note first that any two disjoint subsets of the same level $X_{n}=\mathfrak{c} \times\{n\}$ can be separated by disjoint open sets. To see this, we proceed by induction on $n \epsilon \omega$. Since $X_{0}$ consists of isolated points, we are done for $n=0$. Suppose now that $n \geq 1$, and we are done for $n-1$. Let $A_{0}, A_{1}$ be disjoint subsets of $X_{n}$. Take a $\xi<\lambda$ such that $c_{\xi}(\alpha)=i$, whenever $\langle\alpha, n\rangle \in A_{i}(i \in 2)$. Set $B_{i}=\left\{\langle\beta, n-1\rangle: d_{\xi}(\beta)=i\right\}$. Then for every $i \epsilon 2$ and $x \in A_{i}, N_{x}=\{x\} \cup F(\alpha,\{\xi\}, \emptyset) \times\{n-1\} \in \mathfrak{N}_{x}$ and $N_{x} \subset A_{i} \cup B_{i}$. By our inductive hypothesis, $B_{0}$ and $B_{1}$ can be put into disjoint open subsets $V_{0}$ and $V_{1}$ of $G_{n-1}=\cup_{k \leq n-1} X_{k}$. Then $A_{0} \cup V_{0}$ and $A_{1} \cup V_{1}$ are disjoint open sets separating $A_{0}$ and $A_{1}$.

To prove now that $X$ is hereditarily normal, let $H, K$ be subsets of $X$ such that $\bar{H} \cap K=H \cap \bar{K}=\emptyset$. We have to show that $H$ and $K$ can be separated by disjoint open sets. By the standard shoestring argument, we can assume that $H \subset X_{n}$ for some $n \epsilon \omega$. By passing from $K$ to $K \cup\left(X_{n} \backslash H\right)$, we can also assume that $K \cap X_{n} \cup H=X_{n}$.

Claim 1. If $m \leq n$, then $H$ and $K \cap X_{m}$ can be put into disjoint open subsets.

We have already proved Claim 1 for $m=n$, so suppose $m<n$. Take disjoint open subsets $U$ and $V$ of $G_{m}$ such that $U \supset X_{m} \backslash \bar{K}$ and $V \supset X_{m} \cap \bar{K}$. Then $U^{*}=\left(X \backslash\left(G_{m} \cup \bar{K}\right)\right) \cup U$ and $V^{*}=V$ are disjoint open sets separating $H$ and $K \cap X_{m}$.

By Claim 1, there are disjoint open subsets $W_{H}, W_{K}$ of $G_{n}$ such that $W_{H} \supset H$ and $W_{K} \supset K \cap G_{n}$. Then $W_{H}^{*}=W_{H}$ and $W_{K}^{*}=\left(X \backslash\left(G_{n} \cup \bar{H}\right)\right) \cup W_{K}$ are disjoint open sets separating $H$ and $K$.

Finally, let us start the proof that $X$ is not countably metacompact by defining a subset $Y$ of $\mathfrak{c}$ to be $\sigma$-decomposable if we can find an $f: Y \rightarrow \omega$ and for every $\alpha \epsilon Y$, an $F_{\alpha} \in \mathfrak{F}_{\alpha}$ in such a way that $\alpha \neq \beta$ and $f(\alpha)=f(\beta)$ implies that $\alpha \notin F_{\beta}$ and $\beta \notin F_{\alpha}$. Clearly, every countable union of $\sigma$-decomposable sets is $\sigma$-decomposable and by Lemma $1.2, \mathfrak{c}$ is not $\sigma$-decomposable. Hence, whenever $\mathfrak{c}$ is the union of countably many of its subsets, at least one of those subsets is not $\sigma$-decomposable.

Claim 2. If $n \epsilon \omega$ and $Y \subset \mathfrak{c}$ is not $\sigma$-decomposable, then $Y_{1}=\{\alpha \epsilon Y:\langle\alpha, n+$ 1) $\epsilon \overline{Y \times\{n\}}\}$ is not $\sigma$-decomposable.

To see that Claim 2 is true, let $Y_{0}=Y \backslash Y_{1}$. Since $\alpha \epsilon Y_{0}$ implies that there is an $F_{\alpha} \in \mathfrak{F}_{\alpha}$ with $F_{\alpha} \cap Y=\emptyset, Y_{0}$ is $\sigma$-decomposable (in fact, 1-decomposable). If $Y_{1}$ were now $\sigma$-decomposable, then $Y=Y_{0} \cup Y_{1}$ would be $\sigma$-decomposable, contradicting our assumption.

Passing now to the proof that $X$ is not countably metacompact, consider the increasing open cover $\left\{G_{m}: m \epsilon \omega\right\}$ of $X$. We are going to show that there is no sequence of closed sets $F_{m} \subset G_{m}$ in such a way that $\cup_{m \epsilon \omega} F_{m}=X$. Indeed, if there were such a sequence $\left\langle F_{m}\right\rangle_{m \epsilon \omega}$, then one of the sets $Y_{m}=\left\{\alpha \epsilon \mathfrak{c}:\langle\alpha, 0\rangle \epsilon F_{m}\right\}$ would not be $\sigma$-decomposable. By Claim $2, F_{m} \supset \overline{Y_{m} \times\{0\}}$ would not be a subset of $G_{m}$, contradiction.

Final Remarks. 1. It remains open whether there is a first countable Dowker space in ZFC. The character of spaces similar to our space has to be large if we 
want to carry out the construction in ZFC. Indeed, let us say that $X$ is a nonskipping scattered space of height $\omega$, if $X=\cup_{n \epsilon \omega} X_{n}$, the $X_{n}$ are pairwise disjoint, each $X_{n}$ consists of isolated points of $\cup_{j \geq n} X_{j}$, and whenever $n \epsilon \omega, Y \subset \cup_{k \leq n} X_{n}$ and $\bar{Y} \cap\left(\cup_{j \geq n+1} X_{j}\right) \neq \emptyset$, then $\bar{Y} \cap X_{n+1} \neq \emptyset$. Note that our space is a nonskipping scattered space of height $\omega$. It is easy to see that regular, hereditarily collectionwise Hausdorff, nonskipping scattered spaces of height $\omega$ are paracompact. Since by Fleissner's result $[F]$, every normal space of character $\leq 2^{\omega}$ is collectionwise Hausdorff in the constructible universe, we conclude that under $V=L$, there are no hereditarily normal Dowker spaces of character $\leq 2^{\omega}$ which are nonskipping scattered spaces of height $\omega$.

2. On the other hand, there are consistent examples of spaces with small character similar to the space in this paper. P. deCaux's Dowker space from is of scattered height $\omega$, and it has character $\leq \mathfrak{c}$, because it is locally countable. It is not necessarily hereditarily normal. A deCaux type space of M. E. Rudin $\left[R_{4}\right]$ from $\diamond$ is hereditarily normal, first countable and locally compact, but is neither of scattered height $\omega$ nor $\sigma$-relatively discrete. Dennis Burke and the author observed that an example of S. Shelah [S] can be modified to obtain a consistent example of a locally countable Dowker space (thus of character $\leq \mathfrak{c}$ ) which is both hereditarily normal and is of scattered height $\omega$.

\section{REFERENCES}

[B] Z. Balogh, There is a Q-set space in ZFC, Proc. Amer. Math. Soc. 113 (1991), 557-561. MR 91m:54046

[Be] M. Bell, On the combinatorial principle P(c), Fund. Math. 114 (1981), 137-145. MR 83e:03077

[C] P. deCaux, A collectionwise normal, weakly $\theta$-refinable Dowker space, Topology Proc. 1 (1976), 66-77. MR 56:6629

[D] C. H. Dowker, On countably paracompact spaces, Canad. J. Math. 3 (1951), 219-224. MR 13:264c

[F] W. G. Fleissner, Normal Moore spaces in the constructible universe, Proc. Amer. Math. Soc. 46 (1974), 294-298. MR 50:14682

[G] C. Good, Large cardinals and small Dowker spaces, Proc. Amer. Math. Soc. (to appear)

[JKR] I. Juhasz, K. Kunen, M. E. Rudin, Two more hereditarily separable non-Lindelof spaces, Canad. J. Math. 28 (1976), 998-1005. MR 55:1270

[K] K. Kunen, Set theory, North-Holland, 1980. MR 82f:03001

$[\mathrm{KV}] \mathrm{K}$. Kunen and J. E. Vaughan (eds.), Handbook of set-theoretic topology, North-Holland, 1984. MR 85k:54001

[R $\mathrm{R}_{1}$ M. E. Rudin, Countable paracompactness and Souslin's problem, Canad. J. Math. 7 (1955), 543-547. MR 17:391e

$\left[\mathrm{R}_{2}\right]$ M. E. Rudin, A normal space $X$ for which $X \times I$ is not normal, Fund. Math. 73 (1971), 179-186. MR 45:2660

$\left[\mathrm{R}_{3}\right] \quad$ M. E. Rudin, Two problems of Dowker, Proc. Amer. Math. Soc. 91 (1984), 155-158. MR 85i: $54022 b$

$\left[\mathrm{R}_{4}\right]$ M. E. Rudin, Dowker spaces, in Handbook of set-theoretic topology (K. Kunen and J. E. Vaughan, eds.), North-Holland, 1984, pp. 761-781. MR 86c:54018

$\left[\mathrm{R}_{5}\right] \quad$ M. E. Rudin, A normal screenable nonparacompact space, Topology Appl. 15 (1983), 313322. MR 84d:54042

[R6 $\mathrm{R}_{6}$ M. E. Rudin, Some conjectures, in Open Problems in Topology (J. van Mill and G. M. Reed, eds.) North-Holland, 1990, pp. 183-193. MR 92e:54001

[S] S. Shelah, A consistent counterexample in the theory of collectionwise Hausdorff spaces, Israel J. Mathematics 65 (1989), 219-224. MR 90e:54087

$\left[\mathrm{W}_{1}\right]$ S. Watson, A construction of a Dowker space, Proc. Amer. Math. Soc. 109 (1990), 835-841. MR 91b:54045 
$\left[\mathrm{W}_{2}\right]$ S. Watson, Problems I wish I could solve, in Open Problems in Topology (J. van Mill and G. M. Reed, eds.) North-Holland, 1990, pp. 37-76. MR 92e:54001

[We] W. Weiss, Small Dowker spaces, Pacific J. Math. 94 (1981), 485-492. MR 83d:54036

Department of Mathematics and Statistics, Miami University, Oxford, Ohio 45056

E-mail address: ztbalogh@miavx1.acs.muohio.edu 